\title{
FIRST RESULTS OF THE SPHERE SOLUTION BASED ON HIPPARCOS DATA
}

\author{
L. LINDEGREN ${ }^{1}$, \\ F. van LEEUWEN ${ }^{2}$, C. PETERSEN ${ }^{3}$ and S. SÖDERHJELM ${ }^{1}$ \\ 1 Lund Observatory \\ Box 13, S-22100 Lund, Sweden \\ ${ }^{2}$ Royal Greenwich Observatory \\ Madingley Road, Cambridge CB3 OEZ, U.K. \\ ${ }^{3}$ Copenhagen University Observatory \\ Østervoldgade 3, DK-1350 Copenhagen K, Denmark
}

\begin{abstract}
Results from a test solution of the positions and parallaxes for about 10,000 stars are presented in statistical form. The solution is based on about $\mathbf{1 3 0 0}$ hours of Hipparcos observations made from November 1989 to February 1991, representing about 1/5 of the scientifically useful data collected in that period. Due to the short time span of the data no attempt was made to determine proper motions. The positions, many of which are already in the 2-5 milli-arcsec accuracy range, clearly reveal systematic errors of up to 0.2 arcsec in the ground-based positions of the Input Catalogue. Perhaps the most convincing evidence of the quality of the satellite data is provided by the distribution of trigonometric parallaxes determined in this solution. By comparison with the expected distribution of true parallaxes, based on a standard model of the Galaxy, we conclude that the external errors of the best parallaxes have a standard deviation around 5 milliarcsec. Any global offset of the parallax zero point appears to be less than 1 milli-arcsec. The accuracy obtained is consistent with pre-launch predictions, if allowance is made for the reduced data collection efficiency due to the faulty orbit, and supports the assertion that the 2 milli-arcsec goal can still be reached in the present orbit. Details of the solution are being published in Astronomy 8 Astrophysics.
\end{abstract}

